\title{
THE AUTOMORPHISM GROUP OF A NEAR-RING
}

\author{
S. D. SCOTT
}

\begin{abstract}
As for groups certain automorphisms of a near-ring $\boldsymbol{N}$ may be regarded as inner. The inner automorphisms of $N$ form a normal subgroup of the automorphism group of $N$.

Let $V$ be a group, $\Lambda$ the automorphism group of $V$, and $N$ the near-ring of maps of $V$ into $V$ generated by $\Lambda$. It is shown that if $\Lambda$ is a complete group (see [2, p. 94), then it frequently happens that the automorphisms of $N$ are all inner. Using this result the automorphism groups of certain near-rings may be found.
\end{abstract}

Throughout this paper all near-rings will be left distributive, zero-symmetric and have an identity. If $N$ is a near-ring, then an element of $N$ is a unit if it has a left and a right inverse. The units of $N$ form a group $U(N)$ under composition.

If $\mu$ in $N$ is such that $(\alpha+\beta) \mu=\alpha \mu+\beta \mu$ for all $\alpha$ and $\beta$ in $N$, then we say $\mu$ is distributive. The set of all distributive units of $N$ forms a subgroup of $U(N)$ which we denote by $D(N)$.

A subgroup of $D(N)$ that will be significant in what follows is $Z(N)$. We take $Z(N)$ to be all $\mu$ in $D(N)$ such that $\mu^{-1} \alpha \mu=\alpha$ for all $\alpha$ in $N$. Clearly, if $\lambda$ is in $D(N)$ and $\mu$ is in $Z(N)$, then $\mu^{-1} \lambda \mu=\lambda$ and thus $Z(N)$ is contained in the centre of $D(N)$. It follows that $Z(N)$ is a normal subgroup of $D(N)$.

We shall denote the group of all near-ring automorphisms of $N$ by $A(N)$. If $\mu$ is in $D(N)$ then the map $(\mu) \delta$ of $N$ into $N$ that takes $\alpha$ in $N$ to $\mu^{-1} \alpha \mu$ is in $A(N)$ (such maps are an instance of semilinear maps which have been studied by Ramakotaiah [1, p. 194]). Thus $\delta$ is a map from $D(N)$ into $A(N)$. We denote the image of $\delta$ by $I(N)$. As we shall see in what follows there is an analogy between inner automorphisms of a group and the near-ring automorphisms defined above. Accordingly we call an element of $I(N)$ an inner automorphism of $N$.

Proposition 1. If $N$ is a near-ring then $I(N)$ is a subgroup of $A(N)$ and $I(N) \cong D(N) / Z(N)$.

The proof of this proposition parallels the corresponding proof in group theory.

Let $N$ be a near-ring. The subgroup $I(N)$ of $A(N)$ will be called the group of inner automorphisms of $N$.

Proposition 2. Let $N$ be a near-ring. The subgroup $I(N)$ of $A(N)$ is normal.

Proof. Let $\tau$ be in $A(N)$ and $\mu$ in $D(N)$. Since $\mu \tau$ is in $D(N)$ the proposition will follow if it is shown that $\tau^{-1}(\mu) \delta \tau=(\mu \tau) \delta$. Let $\alpha$ be in $N$. We have

Received by the editors May 21, 1979.

AMS (MOS) subject classifications (1970). Primary 16A76; Secondary 20F28, 16A72. 


$$
\alpha\left[\tau^{-1}(\mu) \delta \tau\right]=\left[\mu^{-1}\left(\alpha \tau^{-1}\right) \mu\right] \tau=\left(\mu^{-1} \tau\right) \alpha(\mu \tau)
$$

and since $\mu^{-1} \tau=(\mu \tau)^{-1}$ it follows that

$$
\alpha\left[\tau^{-1}(\mu) \delta \tau\right]=\alpha[(\mu \tau) \delta] .
$$

Thus $\tau^{-1}(\mu) \delta \tau=(\mu \tau) \delta$ and the proposition holds.

In Theorem 4 we shall be using Proposition 2 to compute the automorphism group of certain near-rings. We first require a further proposition.

Proposition 3. Let $N$ be a near-ring, $\mu$ an element of $D(N)$ and $\tau$ an element of $A(N)$. Assume $Z(N)=\{1\}$. It follows that $(\mu) \delta \tau=\tau(\mu) \delta$ if, and only if, $\mu \tau=\mu$.

Proof. In the proof of Proposition 2 we saw that $\tau^{-1}(\mu) \delta \tau=(\mu \tau) \delta$. Thus if $\mu \tau=\mu$ it follows that $(\mu) \delta \tau=\tau(\mu) \delta$.

Assume $Z(N)=\{1\}$. If $\tau^{-1}(\mu) \delta \tau=(\mu) \delta$ then $(\mu \tau) \delta=(\mu) \delta$ and $(\mu \tau)^{-1} \alpha(\mu \tau)=$ $\mu^{-1} \alpha \mu$ for all $\alpha$ in $N$. Thus

$$
\left[(\mu \tau) \mu^{-1}\right]^{-1} \alpha(\mu \tau) \mu^{-1}=\alpha
$$

for all $\alpha$ in $N$ and, since $(\mu \tau) \mu^{-1}$ is in $D(N)$ and $Z(N)=\{1\}$ it follows that $(\mu \tau) \mu^{-1}=1$. The proposition now follows.

One class of groups of importance to group theory are the complete groups. A group is complete if it has a trivial centre and all group automorphisms are inner (see [2, p. 94]). By analogy with groups we call a near-ring $N$ complete if $I(N)=A(N)$ and $Z(N)=\{1\}$.

THEOREM 4. Let $V$ be an additive group (not necessarily abelian) and let $\Lambda$ be the group of automorphisms of $V$. Let $N$ be the near-ring of maps of $V$ into $V$ generated by $\Lambda$. If $\Lambda$ is a complete group and if there exists $v$ in $V$ such that $v N=V$ then $N$ is a complete near-ring and $A(N) \cong \Lambda$.

Proof. Obviously $\Lambda \subseteq D(N)$. Take $\mu$ in $D(N)$ and let $v$ be as in the statement of the theorem. If $u$ and $w$ are in $V$ then $u=v \alpha$ and $w=v \beta$ where $\alpha$ and $\beta$ are in $N$. Thus

$$
\begin{aligned}
(u+w) \mu & =(v \alpha+v \beta) \mu=v(\alpha+\beta) \mu \\
& =v \delta \mu+v \beta \mu=u \mu+w \mu
\end{aligned}
$$

and we conclude that the $\operatorname{map} \mu$ of $V$ into $V$ is a group homomorphism. Also $N \mu=N$ as $\mu$ is a unit and $v N \mu=v N$. Thus $\mu$ is onto. If $v \alpha \mu=0$ for some $\alpha$ in $N$ then $v \alpha=v \alpha \mu \mu^{-1}=0$ and it follows that $\mu$ is in $\Lambda$. Hence $\Lambda=D(N)$.

Now $I(N) \cong D(N) / Z(N)$ by 1 . Since $\Lambda=D(N), \Lambda$ is a complete group, and $Z(N)$ is contained in the centre of $D(N)$, it follows that $I(N) \simeq \Lambda$.

By $2, I(N)$ is a normal subgroup of $A(N)$ and by [2, p. 94], $I(N)$ is a direct factor of $A(N)$. Thus $A(N)=I(N) \otimes U$ where $U$ is a subgroup of $A(N)$. The theorem will follow if we show that $U$ is trivial.

Let $\tau$ be in $U$. We have $(\mu) \delta \tau=\tau(\mu) \delta$ for all $\mu$ in $D(N)(=\Lambda)$. Since $Z(N)$ is contained in the centre of $D(N)$ which is trivial it follows from 3 that $\mu \tau=\mu$ for all $\mu$ in $D(N)(=\Lambda)$. Now if $\alpha$ is in $N$ then

$$
\alpha=\mu_{1}+\cdots+\mu_{k}
$$


where $-\mu_{i}$ or $\mu_{i}, i=1, \ldots, k$, are in $\Lambda$. Hence

$$
\alpha \tau=\mu_{1} \tau+\cdots+\mu_{k} \tau
$$

and from the above $\mu_{i} \tau=\mu_{i}$ for $i=1, \ldots, k$. It follows that $\alpha \tau=\alpha$ for all $\alpha$ in $N$ and $\tau$ is the identity. Thus $U$ is trivial and the theorem holds.

We state as a corollary a special case of Theorem 4.

COROllary. Let $V$ be an additive group (not necessarily abelian). Let $N$ be the near-ring generated by the inner automorphisms of $V$ and suppose there exists $v$ in $V$ such that $v N=V$. If $V$ is complete then $N$ is a complete near-ring and $A(N) \simeq V$.

EXAMPLES. Let $S_{n}, n>3, n \neq 6$, be the symmetric group on $n$ letters. Suppose $N$ is the near-ring generated by the inner automorphisms of $S_{n}$. Since $S_{n}$ is complete (see [2, p. 96]) and $S_{n}=v N$ for any $v$ not contained in $A_{n}$ (the alternating subgroup) it follows that $A(N) \cong S_{n}$ and $N$ is complete.

The above example is one of many. The holomorph $V$ of a finite cyclic group of odd order is complete (see [2, p. 96]) and there exists a $v$ in $V$ as in the above corollary. Thus for such a group the near-ring $N$ generated by the inner automorphisms of $V$ has the property that it is complete and $A(N) \simeq V$.

Let $V$ be a group and $M_{0}(V)$ the near-ring of all zero-fixing maps of $V$. Ramakotaiah has shown (see [1, p. 196]) that $M_{0}(V)$ is complete and $A\left(M_{0}(V)\right)$ is isomorphic to the automorphism group of $V$.

One question, a group theory question, that arises from the above is which complete groups satisfy the conditions of the above corollary i.e. which complete groups are the normal closure of a single element.

Another question is whether or not this condition is needed in Theorem 4 and the above corollary.

\section{REFERENCES}

1. G. Pilz, Near-rings, North-Holland, Amsterdam, 1977.

2. E. Schenkmann, Group theory, Van Nostrand, Princeton, N.J., 1965.

Department of Mathematics, University of Auckland, Auckinand, New Zealand 